\title{
CHARACTERIZATIONS OF AMENABLE GROUPS
}

\author{
BY \\ WILLIAM R. EMERSON ${ }^{1}$
}

\begin{abstract}
Generalizing a construction of Banach from 1923 we obtain new criteria for the amenability of a locally compact group $G$. The relationship of these new criteria to known characterizations is then investigated, and in particular a formally strenghthened version of the von Neumann/Dixmier condition for amenability is established.
\end{abstract}

0. Introduction and notation. In 1923 Banach [2] showed how to construct translation invariant means on $L_{1}(T)$ (where $T$ is the circle) which do not agree with the Lebesgue integral in general but do agree on the subspace of Riemann integrable functions, and thereby resolved the "Problème de la mesure" which had been open since its proposal in Lebesgue's "Leçons sur l'Intégration" eighteen years earlier. Contained in Banach's construction naturally generalized to an arbitrary locally compact group $G$ there is only one point of possible obstruction, and consequently when isolated and formalized one obtains a condition on $G$ which is readily seen to be equivalent to amenability (in current terminology). It is rather curious that contained implicitly in what is perhaps the first paper on translation invariant means/amenability is a characterization of amenability which has apparently gone unnoticed for over 50 years. The purpose of the present paper is to describe and examine this condition, showing its relationship to known characterizations and to derive further consequences.

In what follows $G$ is a fixed arbitrary locally compact group with a fixed left Haar measure associated, $L_{p}=L_{p}(G)$ are the associated real Lebesgue spaces $(1 \leqslant p \leqslant \infty)$, and $\delta=\delta(G)$ is the real vector space of all real measurable simple functions on $G$. Moreover, for any function $f$ on $G, L_{a} f$ denotes left translation by $a\left(\left(L_{a} f\right)(g)=f(a g)\right)$ and ${ }_{a} \doteqdot L_{a^{-1}} f$. In general the notation follows that of Greenleaf's monograph [3] unless otherwise noted.

I. The basic characterization. The following is essentially the condition used by Banach, appropriately generalized to the present context, which enabled

Received by the editors February 13, 1977.

AMS (MOS) subject classifications (1970). Primary 43A07; Secondary 43A15.

Key words and phrases. Amenable group, invariant mean.

'This work was partially supported by the National Science Foundation Grant MCS 76-06925. 
him to construct a translation invariant mean on $\mathcal{S}=\mathcal{S}(T)$ :

PROPOSITION 1.1. S contains a subspace $\mathfrak{A}$ satisfying the following two properties iff there is a left invariant mean $(\div$ LIM) on $\delta$ :

(i) $L_{g} f-f \in \mathcal{R}$ for all $f \in \mathcal{S}, g \in G$,

(ii) $f \in \mathcal{S}$ and $f>0$ implies $f \notin \Re$.

Comments. Any LIM on $\delta$ clearly has a (unique) extension to a LIM on $L_{\infty}$ and consequently there is a LIM on $\delta$ iff $G$ is amenable. Note also that $f>0$ for $f \in \mathcal{S}$ is trivially equivalent to inf $f=\min f>0$.

PRoof. If $m$ is a LIM on $\mathcal{S}$, let $\Re \div \operatorname{ker}(m)=\{s \in \mathcal{S}: m(s)=0\}$. Then since $m\left(L_{g} f\right)=m(f)$ by the left invariance of $m, m\left(L_{g} f-f\right)=0$ and $L_{g} f-f$ $\in \Re$. Moreover, if $f \in \mathcal{S}$ and $f>0$ then inf $f>0$ and $m(f) \geqslant \inf f>0$ (since $m$ is a mean) and consequently $f \notin \Re$. Conversely, assume there is a subspace $\Re \subseteq \delta$ satisfying (i) and (ii). Rather than follow Banach's original proof via the construction of classes of "hyperfunctions" and the extension of a functional by transfinite induction, we simply observe that (i) and (ii) imply that the von Neumann/Dixmier condition (D) for the amenability of $G[3, \mathrm{p}$. 25] is satisfied:

$$
G \text { is amenable } \Leftrightarrow \Re_{0}=\Re_{0}(G) \doteqdot \text { finite linear span }\left\{L_{8} f-\right.
$$
$f: f \in \mathcal{S}, g \in G\}$ contains no strictly positive function.

(This is not surprising since the "subadditive function" form of the HahnBanach theorem gives the existence of a LIM on $\delta$ immediately from (D)-merely a formalized version of Banach's original proof.) The implication is immediate since (i) gives $\varkappa_{0} \subseteq \Re$ while (ii) guarantees that no strictly positive function is in $\Re$ and, a fortiori, $\varkappa_{0}$. Note that $\varkappa_{0}$ is the minimal candidate for satisfying (i) and (ii) and if $\varkappa_{0}$ violates (ii) no $\supseteq \supseteq \varkappa_{0}$ can possibly satisfy (ii).

Of course Banach did not simply state the prototype of Proposition 1.1; he moreover constructed an $\Re$ (for $G=T$ ) which did in fact satisfy (i) and (ii). We now merely follow his construction, note that there is only one point where there is a possible obstruction (in the general case) and, in the spirit of modern mathematics, hypothesize a criterion which enables us to bypass this obstruction thereby obtaining a condition equivalent to the amenability of $G$. In carrying out this program we first make the following definitions:

Definition 1.2. (a) $P=P(G) \div\left\{\phi \in L_{1}(G): \varphi \geqslant 0,\|\phi\|_{1}=1\right\}$.

(b) $\Re_{P}=\Re_{P}(G) \div\left\{f \in \mathcal{S}: \inf \left\{\|\phi * f\|_{\infty}: \phi \in P\right\}=0\right\}=\{f \in \mathcal{S}: 0$ is in the $\left(L_{\infty}\right.$-norm) closure of $\left.P * f\right\}$, where $*$ denotes convolution.

(c) For any function $f: G \rightarrow R$ define $f^{\sim}$ and $f^{*}$ by $f^{\sim}(x)=f\left(x^{-1}\right)$ and $f^{*}(x)=f\left(x^{-1}\right) \Delta\left(x^{-1}\right)$ for all $x$ in $G$ where $\Delta$ is the modular function.

Comment. In Definition 1.2(b), since $P * f=\{\phi * f: \varphi \in P\}$ is a convex 
subset of $L_{\infty}$, requiring that 0 be in the norm closure of $P * f$ is equivalent to the formally weaker condition that 0 be in the weak closure of $P * f$.

The problem remains of deciding for which $G \Re_{P}(G)$ satisfies the criteria of Proposition 1:

PROPOSITION 1.3. For any locally compact group $G, \Re_{P}(G)$ satisfies criteria (i) and (ii) of Proposition 1 and is closed under scalar multiplication.

Proof. It is easily verified that the mapping $f u \rightarrow f^{*}$ is an isometric involution on $L_{1}(G)$ and also on $P$. Consequently as $\phi$ runs through $P$ so does $\phi^{*}$, and moreover

$$
\begin{aligned}
\left(\phi^{*} * f\right)(x) & =\int_{G} \phi^{*}(t) f\left(t^{-1} x\right) d t=\int_{G} \phi\left(t^{-1}\right) \Delta\left(t^{-1}\right) f\left(t^{-1} x\right) d t \\
& =\int_{G} \phi(t) f(t x) d t .
\end{aligned}
$$

(Note. This formulation enables one to extend Definition 1.2(b) to appropriate semigroups.) First, closure of $\Re_{P}$ under scalar multiplication is trivial since $\|\phi *(c f)\|_{\infty}=|c|\|\phi * f\|_{\infty}$. Moreover, if $f \in \mathcal{S}$ and $f>0$ then $\varepsilon \div \inf f=\min f>0$ and consequently for any $\phi \in P$

$$
\int_{G} \phi(t) f(t x) d t \geqslant \int_{G} \phi(t) \cdot \varepsilon d t=\varepsilon>0,
$$

showing that $\inf \left\{\|\phi * f\|_{\infty}: \phi \in P\right\} \geqslant \varepsilon>0$, verifying (ii). Finally for any fixed $a \in G$ and any subset 0 of $G$ of finite positive measure $|0|$ let

$$
\phi_{n} \doteqdot \frac{1}{n|0|} \sum_{k=1}^{n} \chi_{a^{k_{0}}}
$$

where $\chi_{U}$ is the indicator function of $U$, and consider $f_{n} \doteqdot \phi_{n}^{*} *\left(L_{a} f-f\right)$ :

$$
\begin{aligned}
f_{n}(x) & =\int_{G} \phi_{n}(t)\left(L_{a} f-f\right)(t x) d t=\int_{G} \phi_{n}(t)(f(a t x)-f(t x)) d t \\
& =\frac{1}{n|0|} \sum_{k=1}^{n} \int_{a^{k} 0}(f(a t x)-f(t x)) d t \\
& =\frac{1}{n|0|} \sum_{k=1}^{n}\left(\int_{a^{k} 0} f(a t x) d t-\int_{a^{k} 0} f(t x) d t\right) \\
& =\frac{1}{n|0|}\left(\int_{a^{n+1} 0} f(t x) d t-\int_{a 0} f(t x) d t\right)
\end{aligned}
$$

(upon letting at $\rightarrow t$ in the first integral). Consequently, $\left\|f_{n}\right\|_{\infty}<(2 / n)\|f\|_{\infty} \rightarrow$ 0 as $n \rightarrow+\infty$ and $L_{a} f-f \in \Re_{P}$ and (i) is also verified.

As a consequence of Proposition 1.3, the pivotal issue is identifying those groups $G$ for which $\varkappa_{P}(G)$ is closed under addition since an immediate corollary of Propositions 1.1 and 1.3 is: 
Corollary 1.4. $G$ is amenable if $\Re_{P}(G)$ is closed under addition.

Comment. See Theorem 1.7 for the converse.

The following criterion to insure the closure of $\Re_{P}(G)$ under addition is the one naturally abstracted from Banach's original argument:

THEOREM 1.5. $\Re_{P}(G)$ is closed under addition (and thus $G$ is amenable) if: for any $\phi_{1}$ and $\phi_{2}$ in $P$,

$$
d\left(\phi_{1} * P, \phi_{2} * P\right)=\inf \left\{\left\|\phi_{1} * \hat{\phi}_{1}-\phi_{2} * \hat{\phi}_{2}\right\|_{1}: \hat{\phi}_{1}, \hat{\phi}_{2} \text { in } P\right\}=0 \text {. }
$$

Comments. Criterion (B) states that 0 is in the $L_{1}$-norm closure of the convex set $\phi_{1} * P-\phi_{2} * P$ for any $\phi_{1}$ and $\phi_{2}$ in $P$, and (as in the comment after Definition 1.2(b)) this is equivalent to the formally weaker criterion that 0 be in the weak closure of $\phi_{1} * P-\phi_{2} * P$. Also, see Theorem 1.7 for the converse.

Proof, Fix $f_{1}, f_{2} \in \Re_{P}$ and choose $\varepsilon>0$. Then by the definition of $\Re_{P}$ let $\phi_{i}^{*}=\phi_{i}^{*}\left(f_{i}, \varepsilon\right)(i=1,2)$ satisfy $\left\|\phi_{i}^{*} * f_{i}\right\|_{\infty}<\varepsilon(i=1,2)$. Next choose $\hat{\phi}_{1}$ and $\hat{\phi}_{2}$ in $P$-by (B)-such that $\left\|\phi_{1} * \hat{\phi}_{1}-\phi_{2} * \hat{\phi}_{2}\right\|_{1}<\varepsilon$. Finally, set $\phi_{\varepsilon} \doteqdot \hat{\phi}_{1}^{*} * \phi_{1}^{*}$ $(\in P)$ and consider $\psi_{\varepsilon} \doteqdot \phi_{\varepsilon} *\left(f_{1}+f_{2}\right): \psi_{\varepsilon}=\phi_{\varepsilon} *\left(f_{1}+f_{2}\right)=\phi_{\varepsilon} * f_{1}+\phi_{\varepsilon} * f_{2}$ $=\hat{\phi}_{1}^{*} *\left(\phi_{1}^{*} * f_{1}\right)+\left(\hat{\phi}_{1}^{*} * \phi_{1}^{*}-\hat{\phi}_{2}^{*} * \phi_{2}^{*}\right) * f_{2}+\hat{\phi}_{2}^{*} *\left(\phi_{2}^{*} * f_{2}\right)$ and consequently (since $\|\phi * f\|_{\infty} \leqslant\|\phi\|_{1}\|f\|_{\infty}$ )

$$
\begin{aligned}
\left\|\psi_{\varepsilon}\right\|_{\infty} & \leqslant\left\|\phi_{1}^{*} * f_{1}\right\|_{\infty}+\left\|\hat{\phi}_{1}^{*} * \phi_{1}^{*}-\hat{\phi}_{2}^{*} * \phi_{2}^{*}\right\|_{1}\left\|f_{2}\right\|_{\infty}+\left\|\phi_{2}^{*} * f_{2}\right\|_{\infty} \\
& \leqslant \varepsilon+\varepsilon\left\|f_{2}\right\|_{\infty}+\varepsilon=\varepsilon\left(2+\left\|f_{2}\right\|_{\infty}\right)
\end{aligned}
$$

(the $*$ involution satisfies $(g * h)^{*}=h^{*} * g^{*}$ for $g$ and $h$ in $L_{1}$ and thus $\left.\left\|\hat{\phi}_{1}^{*} * \phi_{1}^{*}-\hat{\phi}_{2}^{*} * \phi_{2}^{*}\right\|_{1}=\left\|\left(\hat{\phi}_{1}^{*} * \phi_{1}^{*}-\hat{\phi}_{2}^{*} * \phi_{2}^{*}\right)^{*}\right\|_{1}=\left\|\phi_{1} * \hat{\phi}_{1}-\phi_{2} * \hat{\phi}_{2}\right\|<\varepsilon\right)$. Upon letting $\varepsilon \downarrow 0$ we conclude that $f_{1}+f_{2} \in \mathcal{T}_{P}$.

Corollary 1.6. Any Abelian group $G$ is amenable.

Proof. Take $\hat{\phi}_{1} \doteqdot \phi_{2}$ and $\hat{\phi}_{2} \doteqdot \phi_{1}$ to verify (B).

It is also easy to prove the converse of Corollary 1.4 and Theorem 1.5 from known characterizations of amenability and we finally obtain the basic result of this paper:

THEOREM 1.7. If $G$ is a locally compact group the following are equivalent:

(a) $G$ is amenable,

(b) $\Re_{P}(G)$ is closed under addition,

(c) $d\left(\phi_{1} * P, \phi_{2} * P\right)=0$ for $\phi_{1}, \phi_{2} \in P$ (criterion (B)).

Proof. (c) $\Rightarrow(b)$ is Theorem 1.5 and (b) $\Rightarrow(a)$ is Corollary 1.4. Moreover, if $G$ is amenable there is a net $\left\{\psi_{\alpha}\right\}$ in $P$ strongly convergent to topological left invariance [3, p. 33], i.e., for any $\phi \in P,\left\|\phi * \psi_{\alpha}-\psi_{\alpha}\right\|_{1} \rightarrow 0$. But this immediately implies that $\left\|\phi_{1} * \psi_{\alpha}-\phi_{2} * \psi_{\alpha}\right\|_{1} \rightarrow 0$ for any $\phi_{1}, \phi_{2} \in P$, which 
in turn trivially shows that $d\left(\phi_{1} * P, \phi_{2} * P\right)=0$. Thus (a) $\Rightarrow$ (c) and the equivalence is complete.

As a consequence of these arguments we have the following result which does not explicitly mention amenability though in fact all the conditions are equivalent to the amenability of $G$ :

Proposition 1.8. If $G$ is a locally compact group and $P=P(G) \doteqdot\{f \in$ $\left.L_{1}(G): f \geqslant 0,\|f\|_{1}=1\right\}$, then the following conditions are equivalent:

(I) For any $\phi_{1}, \phi_{2} \in P, d\left(\phi_{1} * P, \phi_{2} * P\right)=0$ (or equivalently: 0 is in the weak closure of the (convex) set $\phi_{1} * P-\phi_{2} * P$ in $\left.L_{1}\right)$.

(II) For any $\phi_{1}, \phi_{2} \in P, d\left(\left(\phi_{1}-\phi_{2}\right) * P, 0\right)=0$ (or equivalently: 0 is in the weak closure of the (convex) set $\left(\phi_{1}-\phi_{2}\right) * P$ in $\left.L_{1}\right)$.

(III) There is a net $\left\{\psi_{\alpha}\right\}$ in $P$ such that, for any $\phi_{1}, \phi_{2} \in P, \|\left(\phi_{1}-\right.$ $\left.\phi_{2}\right) * \psi_{\alpha} \|_{1} \rightarrow 0$.

(IV) There is a net $\left\{\psi_{\alpha}\right\}$ in $P$ such that, for any $\phi$ in $P,\left\|\phi * \psi_{\alpha}-\psi_{\alpha}\right\|_{1} \rightarrow 0$.

Comment. That (II) $\Rightarrow$ (III) is easy to show directly. It would be of interest to have "direct" proofs of (I) $\Rightarrow$ (II) and (III) $\Rightarrow$ (IV) without interjecting the theory of amenable groups.

Proof. $G$ amenable $\Rightarrow$ (IV) (as referred to in the proof of Theorem 1.7), and (IV) $\Rightarrow(\mathrm{III}) \Rightarrow(\mathrm{II}) \Rightarrow$ (I) are trivial. Finally, that $(\mathrm{I}) \Rightarrow G$ amenable is the main content of Theorem 1.5.

The possibility naturally arises as to considering possibilities for $\Re$ other than $\Re_{P}$ and deriving further criteria for amenability. Interestingly enough, $\Re_{0}$ of condition ( $\left.\mathscr{D}\right)$, the "natural" choice for $\Re$, seems to be intractable. Moreover, consider $\Re_{D} \doteqdot\left\{f \in \delta: \inf \left\{\|d * f\|_{\infty}: d \in D\right\}=0\right\}$ where $D$ is the set of all finite discrete positive measures on $G$ of weight one: since $P * D \subseteq P$ we have $\Re_{D} \subseteq \Re_{P}$ for arbitrary $G$ and it is easy to verify the analogue of Proposition 1.3 for $\Re=\Re_{D}$. But the sufficient criterion for the closure of $\Re_{D}$ under addition that one naturally obtains in this case is not necessary, i.e., condition (B) for $G$ as a discrete group. This approach remains open for further study.

Finally it remains to transfer the "analytic" criterion (B) on $P \subseteq L_{1}$ into a measure theoretic/geometric condition in the group $G$ itself, e.g., the relation between geometric and functional criteria for amenability [3, pp. 64-67]. This program has not been carried out, but the following condition appears to be valid:

Conjecture 1.9. The locally compact group $G$ is amenable $\Leftrightarrow$ if $K_{1}, K_{2}$ are two relatively compact nonempty subsets of $G$ and $\varepsilon>0$, there exist compact sets $C_{1}$ and $C_{2}$ of positive measure such that

$$
\frac{\left|K_{1} C_{1} \Delta K_{2} C_{2}\right|}{\left|C_{1}\right|+\left|C_{2}\right|}<\varepsilon
$$

where $|*|$ denotes a fixed left Haar measure on $G$. 
Comments. Condition (A) $[3$, p. 71$]$ readily implies (R), i.e., the forward implication is valid. Moreover, is there a direct proof that conditions (B) and (R) are equivalent (in case Conjecture 1.9 is valid)?

II. Consequences and relations to prior results. In this section we first derive the "Localization Theorem" [5] (in its most important case of $L_{\infty}(G)$ ) from Proposition 1.8(II). We will need the following variant of the Hahn-Banach theorem:

Proposition 2.1. If $N$ is a subspace of the real vector space $L_{\infty}=L_{\infty}(X, \mu)$ then there is a mean $m$ in $L_{\infty}^{*}$ such that $m(N)=0 \Leftrightarrow$ ess inf $g<0$ for all $g$ in $N$.

Proof. If ess inf $g_{0}>0$ then by the definition of a mean $m\left(g_{0}\right) \geqslant$ ess inf $g_{0}>0$ and consequently $g_{0} \notin N$. Conversely, let $p(g)=$ ess sup $g$ for $g$ in $L_{\infty}$ and $L$ be the zero functional on $N$. Then by a standard form of Hahn-Banach [4, pp. 434-435] $L$ may be extended to a functional $m$ in $L_{\infty}^{*}$ dominated by $P$, and domination by $p$ readily implies, moreover, that this $m$ must in fact be a mean.

We also need this result in the following special context:

COROllary 2.2. If $f$ is in $L_{\infty}(G)$, then there is a mean $m_{f}$ in $L_{\infty}^{*}$ such that $m_{f}(\phi * f)=m_{f}(f)$ for all $\phi$ in $P$ iff inf $\left(\phi_{1}-\phi_{2}\right) * f<0$ for all $\phi_{1}, \phi_{2}$ in $P$.

Proof. By 2.1 such an $m_{f}$ exists iff ess inf $g<0$ for all $g$ in $N \div$ finite linear span $\{\phi * f-f: \phi$ in $P\}$. Since $\left(\phi_{1}-\phi_{2}\right) * f=\left(\phi_{1} * f-f\right)-\left(\phi_{2} * f\right.$ $-f$ ) (and $\left(\phi_{1}-\phi_{2}\right) * f$ is continuous allowing us to replace ess inf by inf) is clearly in $N$ one implication is clear. On the other hand, if $\eta=\Sigma a_{i}\left(\phi^{i} * f-\right.$ $f$ ) in $N$ satisfies ess inf $\eta=\varepsilon>0$, then for any $\phi$ in $P$ inf $\phi * \eta \geqslant \varepsilon>0$ also. But $\phi * \eta=\Sigma a_{i}\left(\psi_{i} * f-\phi * f\right)$ where $\psi_{i}=\phi * \phi^{i}$ is in $P$, and upon setting $A \div \Sigma\left|a_{i}\right|$ it is easy to see that $A^{-1}(\phi * \eta)(\geqslant \varepsilon / A>0)$ is of the form $\left(\phi_{1}-\phi_{2}\right) * f$ for appropriate $\phi_{1}, \phi_{2}$ in $P$ and the converse implication is verified.

We are now prepared to prove:

THEOREM 2.3. Let $G$ be a locally compact group. Then for each $f$ in $L_{\infty}(G)$ there is a mean $m_{f}$ in $L_{\infty}^{*}$ such that $m_{f}(\phi * f)=m_{f}(f)$ for all $\phi$ in $P$ iff there is a mean $m$ in $L_{\infty}^{*}$ such that $m(\phi * f)=m(f)$ for all $\phi$ in $P$ and $f$ in $L$, i.e., there exists a TLIM (topological left invariant mean) $m$ on $L_{\infty}(G)$.

Comments. The existence of a TLIM on $L_{\infty}(G)$ is known to be equivalent to the amenability of $G[3$, p. 26]. Theorem 2.3 is the "local" characterization of the amenability of $G$ as shown in [5] by use of the Arens product [1].

Proof. It suffices to show that the existence of the $m_{f}$ for all $f$ in $L_{\infty}$ forces the validity of $1.8(\mathrm{II})$. To this end fix any $\phi_{1}, \phi_{2}$ in $P$ and consider $N \doteqdot\left(\phi_{1}^{*}-\right.$ $\left.\phi_{2}^{*}\right) * L_{\infty}=\left\{\left(\phi_{1}^{*}-\phi_{2}^{*}\right) * f: f \in L_{\infty}\right\}$ (see 1.2(c)): $N$ is clearly a subspace of 
$L_{\infty}$. For any $f$ in $L_{\infty}$ since $m_{f}$ exists, by 2.2 we must have inf $\left(\phi_{1}^{*}-\phi_{2}^{*}\right) * f<$ 0 , and consequently by 2.1 there is a mean $m$ in $L_{\infty}^{*}$ such that $m(N)=0$. Since $P$ is weak-* dense in the set of means in $L_{\infty}^{*}\left[3\right.$, p. 22] there is a net $\left\{\psi_{\alpha}\right\}$ in $P$ such that $\left(\psi_{\alpha}, h\right) \rightarrow m(h)$ for all $h$ in $L_{\infty}$. In particular for $h=\left(\phi_{1}^{*}-\right.$ $\left.\phi_{2}^{*}\right) * f$ in $N$ we have $\left(\psi_{\alpha}, h\right)=\left(\psi_{\alpha},\left(\phi_{1}^{*}-\phi_{2}^{*}\right) * f\right)=\left(\left(\phi_{1}-\phi_{2}\right) * \psi_{\alpha}, f\right) \rightarrow$ $m(h)=0$, where we have used $\left(\psi, \phi^{*} * f\right)=(\phi * \psi, f)$ for $\psi, \phi$ in $L_{1}$ and $f$ in $L_{\infty}$-see [5, Lemma 3.1]. But this is precisely 1.8 (II) -0 is in the weak closure of $\left(\phi_{1}-\phi_{2}\right) * P$-and the demonstration is completed.

The idea used in the proof of 2.3 may be extended, and to that end we make the following

Definition 2.4. Let $G$ be a locally compact group. The subset $X \subseteq L_{\infty}(G)$ is said to be full (in $L_{\infty}(G)$ ) iff for every $\phi_{1}, \phi_{2}$ in $P(G)$ the following is true: there is a net $\left\{\psi_{\alpha}\right\}=\left\{\psi_{\alpha}\left(\phi_{1}, \phi_{2}\right)\right\}$ in $P$ such that $\left(\left(\phi_{1}-\phi_{2}\right) * \psi_{\alpha}, f\right) \rightarrow 0$ for all $f$ in $X$ iff 0 is in the $L_{1}$ norm closure (or equivalently the weak closure) of the convex set $\left(\phi_{1}-\phi_{2}\right) * P \subseteq L_{1}$.

Comment. If $X$ is full and $X \subseteq \hat{X}$ then trivially $\hat{X}$ is also full. The proof of 2.3 used that $X=L_{\infty}(G)$ is full for any $G$.

In case $X$ is a subspace of $L_{\infty}$ we may use a formally weakened form of 2.4 in defining full subspace as a consequence of

Proposition 2.5. Let $X$ be a real vector space, $X^{*}$ the (real algebraic) dual of $X$, and $C$ a convex subset of $X^{*}$. Then $f$ in $X^{*}$ is weak-* adherent to $C$, i.e., there is a net $\left\{f_{\alpha}\right\}$ in $C$ such that $\left(f_{\alpha}, x\right) \rightarrow(f, x)$ for all $x$ in $X$ iff for each $x$ in $X$ there is a net-therefore a sequence- $\left\{f_{n}(x)\right\}$ in $C$ such that $\left(f_{n}(x), x\right) \rightarrow$ $(f, x)$.

Proof. Without loss of generality assume $f=0$ (by replacing $C$ by $C-f$, etc.) and fix any $x_{1}, \ldots, x_{k}$ in $X$. Consider the linear mapping $T: X^{*} \rightarrow R^{k}$ defined by $f \rightarrow T(f) \div\left(\left(f, x_{1}\right), \ldots,\left(f, x_{k}\right)\right)$. First, $S \div T(C)$ is a convex subset of $R^{k}$ (since $C$ is convex in $X^{*}$ ). Showing that there is a net $\left\{f_{\alpha}\right\}$ in $C$ converging weakly to 0 is now equivalent to showing that 0 is in the closure $\bar{S}$ of $S$ in $R^{k}$ (for any choice of finite subset $x_{1}, \ldots, x_{k}$ in $X$ ). Proceeding by contradiction, if 0 is not in $\bar{S}$ there is a hyperplane separating 0 from the convex set $\bar{S}$ or equivalently a vector $A=\left(a_{1}, \ldots, a_{k}\right)$ in $R^{k}$ such that $A \cdot s>1$ for all $s$ in $\bar{S}$. Consider $x_{0} \doteqdot a_{1} x_{1}+\cdots+a_{k} x_{k}$ in $X$ :

Let $\left\{f_{n}\left(x_{0}\right)\right\}$ be a sequence in $C$ such that $\left(f_{n}\left(x_{0}\right), x_{0}\right) \rightarrow 0$, and choose $f_{0}$ in this sequence such that $\left(f_{0}, x_{0}\right)<1$. Then $s_{0} \div T\left(f_{0}\right)$ is in $S$ and consequently

$$
\begin{aligned}
A \cdot s_{0} & =\left(a_{1}, \ldots, a_{k}\right) \cdot\left(\left(f_{0}, x_{1}\right), \ldots,\left(f_{0}, x_{k}\right)\right) \\
& =a_{1}\left(f_{0}, x_{1}\right)+\cdots+a_{k}\left(f_{0}, x_{k}\right)=\left(f_{0}, a_{1} x_{1}+\cdots+a_{k} x_{k}\right) \\
& =\left(f_{0}, x_{0}\right)<1 .
\end{aligned}
$$


Corollary 2.6. If $X$ is a subspace of $L_{\infty}(G)$ then there is a net $\left\{\psi_{\alpha}\right\}=$ $\left\{\psi_{\alpha}\left(\phi_{1}, \phi_{2}\right)\right\}$ in $P$ such that $\left(\left(\phi_{1}-\phi_{2}\right) * \psi_{\alpha}, f\right) \rightarrow 0$ for all $f$ in $X \Leftrightarrow$ for each $f$ in $X$ there is a sequence $\psi_{n}(f)=\psi_{n}\left(f, \phi_{1}, \phi_{2}\right)$ in P such that $\left(\left(\phi_{1}-\phi_{2}\right) * \psi_{n}(f), f\right)$ $\rightarrow 0$.

Proof. Apply Proposition 2.5 with $C \doteqdot\left(\phi_{1}-\phi_{2}\right) * P$ and $f=0$.

THEOREM 2.7. If $X$ is a full subspace of $L_{\infty}(G)$ then the following are equivalent:

(1) $G$ is amenable.

(2) There is a mean $m$ on $L_{\infty}(G)$ such that $m(\phi * f)=m(f)$ for all $\phi$ in $P$ and $f$ in $X$.

(3) For each $f$ in $X$ there is a mean $m_{f}$ on $L_{\infty}(G)$ such that $m_{f}(\phi * f)=m_{f}(f)$ for all $\phi$ in $P$.

Comment. If $X$ is a topologically left invariant subspace of $L_{\infty}(P * X \subseteq$ $X$ ) then (2) is equivalent to the existence of a TLIM on $X$ (any mean on any subspace may be extended to a mean on $L_{\infty}$ ), and in particular the theorem states that $L_{\infty}(G)$ supports a TLIM iff $X$ does (in general a TLIM on a subspace cannot be extended to a TLIM on $L_{\infty}$, only to a mean on $L_{\infty}$ ).

PROOF. (1) $\Rightarrow$ (2) by taking $m$ to be any TLIM on $L_{\infty}(G)$, and (2) $\Rightarrow$ (3) by taking $m_{f}=m$. To see that $(3) \Rightarrow(1)$ we follow the proof of 2.3 and first observe by 2.2 that we have $\inf \left(\phi_{1}^{*}-\phi_{2}^{*}\right) * f \leqslant 0$ for all $f$ in $X$ and $\phi_{1}, \phi_{2}$ in $P$. Fixing any $\phi_{1}, \phi_{2}$ in $P$ and setting $N \doteqdot\left(\phi_{1}^{*}-\phi_{2}^{*}\right) * X$, let $m$ be the mean guaranteed by 2.1 and $\left\{\psi_{\alpha}\right\}$ a net in $P$ weak-* convergent to $m$. Then in particular for all $h$ in $N$ we have $\left(\psi_{\alpha}, h\right) \rightarrow m(h)=0$ implying $\left(\psi_{\alpha},\left(\phi_{1}^{*}-\right.\right.$ $\left.\left.\phi_{2}^{*}\right) * f\right)=\left(\left(\phi_{1}^{*}-\phi_{2}^{*}\right)^{*} * \psi_{\alpha}, f\right)=\left(\left(\phi_{1}-\phi_{2}\right) * \psi_{\alpha}, f\right) \rightarrow 0$ for all $f$ in $X$. Since $X$ is full, by definition we conclude that 0 is in the $L_{1}$-norm closure of $\left(\phi_{1}-\phi_{2}\right) * P$. Thus $1.8(\mathrm{II})$ is verified and $G$ is amenable.

The following is a sufficient condition for $X$ to be full.

PRoposition 2.8. $X$ is a full subspace of $L_{\infty}(G)$ if for each $h$ in $L_{\infty}(G)$ there are two nets $\left\{\varepsilon_{\beta}\right\},\left\{f_{\beta}\right\}$ in $P$ (possibly depending on $h$ ) such that $\varepsilon_{\beta} * h * f_{\beta}^{\sim}$ is in $X$ for all $\beta$ and $\left\{\varepsilon_{\beta}\right\}$ is an approximate identity for $L_{1}(G)$.

Proof. Assume $\left(\left(\phi_{1}-\phi_{2}\right) * \psi_{\alpha}, f\right) \rightarrow 0$ for all $f$ in $X$. Then given any $f$ in $X$ and $\omega>0$ there is a $\psi_{\omega}=\psi_{\omega}(f)$ in $P$ such that $\left|\left(\phi * \psi_{\omega}, f\right)\right|<\omega$, where we set $\phi \doteqdot \phi_{1}-\phi_{2}$ for brevity. In particular for any $h$ in $L_{\infty}$ and corresponding $\left\{\varepsilon_{\beta}\right\},\left\{f_{\beta}\right\}$, for each $f=\varepsilon_{\beta} * h * f_{\beta}^{\sim}$ in $X$ there is a $\psi_{\omega}=\psi_{\omega}(f)=\psi_{\omega}(h, \beta)$ in $P$ such that

$$
\left|\left(\phi * \psi_{\omega}, f\right)\right|=\left|\left(\phi * \psi_{\omega}, \varepsilon_{\beta} * h * f_{\beta}^{\sim}\right)\right|=\left|\left(\varepsilon_{\beta}^{*} * \phi *\left(\psi_{\omega} * f_{\beta}\right), h\right)\right|<\omega .
$$

Next, since $\left\{\varepsilon_{\beta}\right\}$ is an approximate identity so is $\left\{\varepsilon_{\beta}^{*}\right\}$ [5, Lemma 3.3] and thus for appropriate $\beta=\beta_{0}$ we have $\left\|\phi-\varepsilon_{\beta_{0}}^{*} * \phi\right\|_{1}<\omega$. Consequently upon 
setting $\hat{\psi}_{\omega}=\psi_{\omega}\left(h, \beta_{0}\right) * f_{\beta_{0}}$ and $\hat{\varepsilon}=\varepsilon_{\beta_{0}}^{*}$ we obtain

$$
\begin{aligned}
\left|\left(\phi * \hat{\psi}_{\omega}, h\right)\right| & \leqslant\left|\left(\hat{\varepsilon} * \phi * \hat{\psi}_{\omega}, h\right)\right|+\left|\left((\phi-\hat{\varepsilon} * \phi) * \hat{\psi}_{\omega}, h\right)\right| \\
& <\omega+\left\|(\phi-\hat{\varepsilon} * \phi) * \hat{\psi}_{\omega}\right\|_{1}\|h\|_{\infty}<\omega+\omega\|h\|_{\infty} .
\end{aligned}
$$

Therefore for each $h$ in $L_{\infty}(G)$ there is a sequence $\hat{\psi}_{k}=\hat{\psi}_{k}(h)$ in $P$ (simply take $\left.\omega=\omega_{k} \downarrow 0, \hat{\psi}_{k} \div \hat{\psi}_{\omega_{k}}\right)$ such that $\left(\phi * \hat{\psi}_{k}, h\right)=\left(\left(\phi_{1}-\phi_{2}\right) * \hat{\psi}_{k}, h\right) \rightarrow 0$. Consequently by Corollary 2.6 there is a net in $\left(\phi_{1}-\phi_{2}\right) * P$ weakly convergent to 0 and thus, by Definition $2.4, X$ is full.

An immediate consequence of 2.8 is

COROLlaRY 2.9. $\mathrm{UCB}(G)$ is a full (topologically left invariant) subspace of $L_{\infty}(G)$ for any locally compact group $G$. Moreover, $G$ is amenable iff there is a TLIM on $\mathrm{UCB}(G)$.

Comment. This result is well known [3, pp. 26-27].

Proof. $\varepsilon * h * \delta^{\sim}$ is in $\operatorname{UCB}(G)$ for arbitrary $\varepsilon$ and $\delta$ in $L_{1}(G)$ and $h$ in $L_{\infty}(G)$.

We conclude this paper with a strengthened form of the von Neumann/ Dixmier criterion (D) for amenability. We first show:

Proposition 2.10. If $X$ is a full subspace of $L_{\infty}(G)$ then $\inf \left(\phi_{1}-\phi_{2}\right) * f \leqslant 0$ for all $\phi_{1}, \phi_{2}$ in $P$ and $f$ in $X$ iff $G$ is amenable.

Proposition 2.10A (CONTRAPOSITIVE FORM). If $X$ is a full subspace of $L_{\infty}(G)$ then $G$ is not amenable iff there exists $\phi_{1}, \phi_{2}$ in $P$ and $f$ in $X$ such that $\inf \left(\phi_{1}-\phi_{2}\right) * f \geqslant 1$.

Proof. Proposition 2.10 is immediate from 2.2 and 2.7, whereas $2.10 \mathrm{~A}$ follows upon also observing that $\inf \left(\phi_{1}-\phi_{2}\right) * f>0 \operatorname{implies} \inf \left(\phi_{1}-\right.$ $\left.\phi_{2}\right) *(c f)>1$ for sufficiently large positive scalars $c$.

We also need the following simple technical

LemMa 2.11. Let $G$ be a locally compact group. For any measurable subset $A$ of finite positive measure $|A|$ let $\phi_{A} \doteqdot \chi_{4} /|A|$, the normalized characteristic function of $A$, and for any $\tau$ in $G$ let $\delta_{\tau}$ denote the Dirac measure (point mass) at $\tau$. Then given any neighborhood $U$ of the identity in $G$ the set $S_{U}$ of simple functions is $\left(L_{1}\right.$-norm) dense in $P$ where

$S_{U} \doteqdot\left\{\frac{1}{n} \sum_{i=1}^{n} \delta_{\tau_{i}} * \phi_{U_{i}}: n\right.$ a positive integer, $\tau_{i}$ in $G$ and $U_{i} \subset U$ for $\left.1 \leqslant i \leqslant n\right\}$.

Proof. The set of simple functions 


$$
S \doteqdot\left\{\begin{array}{l}
\sum_{i=1}^{N} \alpha_{i} \chi_{K_{i}}: N \text { a positive integer, } K_{i} \text { relatively } \\
\text { compact and } \alpha_{i}>0 \text { for } 1 \leqslant i \leqslant n \text { and } \\
\alpha_{1}\left|K_{1}\right|+\cdots+\alpha_{N}\left|K_{N}\right|=1
\end{array}\right\}
$$

is, of course, dense in $P$. Given a neighborhood $U$ of the identity we may cover any relatively compact set $K$ by finitely many left translates of $U$ and this yields a partitioning of $K$ into sets of the form $\tau U^{\prime}$ where $U^{\prime} \subseteq U$ (if $K \subseteq \cup \tau_{i} U$ define $U_{1}^{\prime}$ by $\tau_{1} U_{1}^{\prime} \doteqdot \tau_{1} U \cap K$ and recursively $U_{j}^{\prime}$ by $\tau_{j} U_{j}^{\prime} \doteqdot \tau_{j} U$ $\cap K-\cup\left\{\tau_{r} U: r<j\right\}$ ). Consequently in the definition of $S$ we may assume without loss of generality that each $K_{i}$ is in fact of the form $\tau U^{\prime}$ for some $U^{\prime} \subseteq U$ and also, since sets of measure zero do not "change" $L_{1}$ functions, that each $\left|K_{i}\right|>0$. Now consider any $f$ in $S$ so represented:

$$
f=\sum_{i=1}^{N} \alpha_{i} \chi_{\pi_{i} U_{i}} \quad\left(U_{i} \subseteq U, \alpha_{i}\left|U_{i}\right|>0, \sum_{i=1}^{N} \alpha_{i}\left|U_{i}\right|=1\right) .
$$

Then $f$ may be rewritten as $\sum_{i=1}^{N} \beta_{i} \phi_{\tau_{i}} U_{i}$ where $\beta_{i}=\alpha_{i}\left|U_{i}\right|>0$ and $\sum_{i=1}^{N} \beta_{i}=$ 1. It is also clear that by restricting all $\beta_{i}$ to be fractions in such a representation one obtains a subfamily $S_{U}$ of $S$ dense in $S$ and consequently in $P$. To see that $S_{U}$ may be represented as in the lemma, consider $f=$ $\sum_{i=1}^{N} r_{i} \phi_{\tau_{i}}$ with all $r_{i}>0$ rational and $\sum_{i=1}^{N} r_{i}=1$, the generic function in $S_{U}$. Since $\phi_{\tau U}=\delta_{\tau} * \phi_{U},(1 / n) \sum_{i=1}^{n} \delta_{\tau_{i}} * \phi_{U_{i}}=\sum_{i=1}^{n}(1 / n) \phi_{\tau_{i} U_{i}}$ is in $S_{U}$. Conversely, if $n$ is a common denominator for the $r_{i}$, say $r_{i}=n_{i} / n$, then

$$
\sum_{i=1}^{N} r_{i} \phi_{\tau_{i} U_{i}}=\frac{1}{n} \sum_{i=1}^{N} n_{i}\left(\delta_{\tau_{i}} * \phi_{U_{i}}\right)=\frac{1}{n} \sum_{j=1}^{n} \delta_{\tilde{\tau}_{j}} * \phi_{\hat{U}_{j}}
$$

where each $\delta_{\tau_{i}} * \phi_{U_{i}}$ is merely used $n_{i}$ times as a $\delta_{i_{j}} * \phi_{\dot{U}_{j}}\left(\sum_{i=1}^{N} n_{i}=n\right)$.

We are now in a position to prove

THEOREM 2.12. The locally compact group $G$ is not amenable iff there is an $f$ in $\mathrm{UCB}(G)$ and elements $r_{i}, s_{i}(1 \leqslant i \leqslant n)$ in $G$ such that

$$
\sum_{i=1}^{n}\left({ }_{r_{i}} f-{ }_{s_{i}} f\right) \geqslant 1 \text {. }
$$

Comment. The von Neumann/Dixmier condition in its contrapositive formulation is essentially of the form (C) except that there are allowed different functions $f_{i}$ for each value of the index $i$.

Proof. One implication is trivial since $m$ a LIM on $L_{\infty}$ and $f$ any function in $L_{\infty}$ satisfying (C) gives a contradiction upon applying $m$ to both sides of (C). Conversely if $G$ is not amenable upon applying $2.10 \mathrm{~A}$ to $X=\mathrm{UCB}(G)$ we obtain an $f$ in $\operatorname{UCB}(G)$ and $\phi_{1}, \phi_{2}$ in $P$ such that $\left(\phi_{1}-\phi_{2}\right) * f \geqslant 1$. 
Essentially this same $f$ will be shown to satisfy (C), i.e., any scalar multiple $c f$ of $f$ with $c>1$ will suffice. To this end, we first note that given $f$ in $\operatorname{UCB}(G)$ and $\varepsilon>0$ there corresponds a neighborhood $U=U(f, \varepsilon)$ of the identity such that $\left\|\phi_{U^{\prime}} * f-f\right\|_{\infty}<\varepsilon$ for any measurable $U^{\prime} \subseteq U$ with $\left|U^{\prime}\right|>0$ (this result uses only the right uniform continuity of $f$ and is immediate from the definition). Next, if $D_{i}$ in $S_{U}$ approximates $\phi_{i}(i=1,2)$ in $L_{1}$ to within $\varepsilon$ then

$$
\begin{gathered}
\left\|\left(D_{1}-D_{2}\right) * f-\left(\phi_{1}-\phi_{2}\right) * f\right\|_{\infty} \leqslant\left\|\left(D_{1}-\phi_{1}\right) * f\right\|_{\infty}+\left\|\left(D_{2}-\phi_{2}\right) * f\right\|_{\infty} \\
<\left\|D_{1}-\phi_{1}\right\|_{1}\|f\|_{\infty}+\left\|D_{2}-\phi_{2}\right\|_{1}\|f\|_{\infty} \leqslant 2 \varepsilon\|f\|_{\infty},
\end{gathered}
$$

implying

$$
\left(D_{1}-D_{2}\right) * f \geqslant\left(\phi_{1}-\phi_{2}\right) * f-2 \varepsilon\|f\|_{\infty} .
$$

Moreover, if $D_{1}=(1 / n) \sum_{i=1}^{n} \delta_{r_{i}} * \phi_{U_{i}}$ then

$$
\begin{aligned}
\left\|D_{1} * f-\frac{1}{n} \sum_{i=1}^{n}{ }_{r_{i}} f\right\|_{\infty} & =\left\|\frac{1}{n} \sum_{i=1}^{n} \delta_{r_{i}} *\left(\phi_{U_{i}} * f-f\right)\right\|_{\infty} \\
& <\frac{1}{n} \sum_{i=1}^{n}\left\|\phi_{U_{i}} * f-f\right\|_{\infty}<\varepsilon .
\end{aligned}
$$

Similarly, since we may assume that $n$ is the same in the representations of both $D_{1}$ and $D_{2}$ by being "redundant" if necessary, if $D_{2}=(1 / n) \sum_{i=1}^{n} \delta_{s_{i}} * \phi_{U_{i}^{\prime}}$ then $\left\|D_{2} * f-(1 / n) \sum_{i=1}^{n} f\right\|_{\infty}<\varepsilon$. Thus we finally obtain

$$
\frac{1}{n} \sum_{i=1}^{n}\left({ }_{r_{i}} f-{ }_{s_{i}} f\right) \geqslant\left(D_{1}-D_{2}\right) * f-2 \varepsilon \geqslant\left(\phi_{1}-\phi_{2}\right) * f-2 \varepsilon\left(1+\|f\|_{\infty}\right) \text {. }
$$

Consequently if $\left(\phi_{1}-\phi_{2}\right) * f \geqslant 1$, for any $c>1$ taking $\varepsilon$ small enough one can find $r_{i}, s_{i}$ such that

$$
\frac{1}{n} \sum_{i=1}^{n}\left({ }_{r_{i}} f-{ }_{s_{i}} f\right)>1 / c
$$

and the function ( $c f$ satisfies (C).

Comment. The proof uses that $f$ is in $\operatorname{UCB}(G)$-or more precisely that $f$ is right uniformly continuous-in a crucial sense, the reason being that if one only knows $\left(\phi_{1}-\phi_{2}\right) * f>1$ for a general function $f$ in $L_{\infty}$ there appears to be no way to obtain an analogous inequality involving left translates of $f$. A slight extension of the ideas in the proof of 2.12 yields: if $\mathrm{UCB}_{r}(G)$ is the space of (real) right uniformly continuous bounded functions on $G$ and measures $\mu$ in $M^{+}(G)$ are considered as operators on $\operatorname{UCB}_{r}(G)$ via $\mu(f) \div$ $\mu * f$, then the finite discrete measures $\left\{(1 / n) \sum_{i=1}^{n} \delta_{\tau_{i}}: n\right.$ a positive integer, $\tau_{i}$ in $G, 1<i \leqslant n\}$ are strong operator dense in the set of all positive measures of mass one.

The failure of this density theorem for $M^{+}(G)$ operating on $L_{\infty}(G)$ is why 
we had to work in a smaller subspace $(\operatorname{UCB}(G)$ being chosen for convenience).

In conclusion, the extension of these results to amenable semigroups and group/semigroup actions does not seem unreasonable. Also in §II no use was made of Proposition 1.8(I), the "weakest" criterion for amenability. It is to be hoped that by using this stronger characterization one may obtain a strengthened version of Theorem 2.12. The author wishes to thank Dr. F. P. Greenleaf for many stimulating discussions relating to the material presented in this article.

\section{BIBLIOGRAPHY}

1. R. Arens, The adjoint of a bilinear operator, Proc. Amer. Math. Soc. 2 (1951), 839-848.

2. S. Banach, Sur le probleme de la mesure, Fund. Math. 4 (1923), 7-31.

3. F. P. Greenleaf, Invariant means on topological groups and their applications, Van Nostrand Math. Studies, No. 16, Van Nostrand, New York, 1969.

4. E. Hewitt and K. A. Ross, Abstract harmonic analysis. I, Academic Press, New York, 1963.

5. J. C. S. Wong, Topologically stationary locally compact groups and amenability, Trans. Amer. Math. Soc. 144 (1969), 351-363.

Department of Mathematics, Queens College, CUNY, Flushing, New York 11367 